\title{
HIGH-GAIN FREE ELECTRON LASERS AS GENERATORS \\ OF SHORT WAVELENGTH COHERENT RADIATION*
}

\author{
Kwang-Je Kim \\ Lawrence Berkeley Laboratory \\ University of California, \\ Berkeley, California 94720 \\ Claudio Pellegrini \\ LBL -21562 \\ DE86 012500 \\ Brookhaven National Laboratory \\ Upton, New York 11973
}

\begin{abstract}
The development of coherent radiation in high-gain free electron lasers, either from initial noise or from low-power input radiation, is analyzed in terms of three-dimensional Maxwell-Klimontovich equations. Exponential growth and saturation, transverse radiation profiles, transverse coherence and spectral features are discussed. Two possible systems of high-gain free electron lasers, one based on a storage ring and by-pass, another based on a linac and damping ring, are considered for the generation of $400 \AA$ radiation.
\end{abstract}

\section{INTRODUCTION}

High-gain free electron lasers (FELs) are potentially important as generators of intense, coherent raciation at wavelengths shorter than $1000 \AA, 1$ a region difficult for atomic lasers. Two notes of operations are possible: In the amplifier mode, coherent input radiation, if available at the desired wavelengths, is am.plified to high intensity. In the so-called self-amplified spontaneous emission (SASE) mode, the noise present in the beam is self-amplified to produce high-power coherent radiation. The high-gain FELs thus eliminate the need for the optical cavities required by FEL oscillators. Highgain FELs will deliver higher peak power with lower repetition rate and thus complementary to FEL oscillators. At microwave wavelengths, the principle of the high-gain FEis has been tested experimentally at Lawrence Livermore National Laboratory. 2 In this paper, we discuss general characteristics of high-gain FELs, in particular the properties of SASE. We also evaluate possible.high-gain FEL systems as sources of short wavelength coherent radiation.

*This work was supported by the Director, Office of Energy Research, Office of High Energy and Nuclear Physics, High Energy Physics Division, U.S. Department of Energy under Contract No. DE-AC03-76SF00098, and, in part, under Brookhaven National Laboratory DOE Contract No. DE-ACO2-76CHOOO16. 
The noise in the electron current is due to the discreteness of electrons. The radiation generated by the noise current in undulators, familiar as the undulator radiation in synchrotron radiation research, 3 is analogous to the spontaneous emission in atomic systems. The name SASE derives from this analogy. The characteristics of the undulator radiation is well understood. Due to the self-reinforcing nature of the amplification process, the characteristics of SASE are expected to differ from those of undulator radiation.

In section (II), we give an outline of a consistent threedimensional analysis of the high-gain process. The characteristics of high-gain FELs derived from this analysis are summarized in the following two sections, in section (III) transverse characteristics and intensity characteristics in section (IV). Section (V) discusses a high-gain FEL for $400 \AA$ based on an electron storage ring and bypass. In section (VI), an alterrative system based on a linac and a damping ring is considered.

\section{METHOD OF ANALYSIS}

The high-gain behavior of FEL amplifier in one-dimensional theory is well-known. 4 Important aspects of SASE have been derived from intuitive analysis. 1 Recently, a one-dimensional theory of SASE was developed based on the coupled Maxwell-Klimontovich equations. 5 The theory was further extended to include three-dimensional effects, 6 which are important for a correct understanding of high-gain properties. Here we give an outline of the analysis given in Ref. (6).

We choose $z$, the distance from the undulator entrance, as the independent variable. The transverse coordinates are given by a twodimensional vector $x$. The dynamical variables describing the electron motion are the phase $\theta$ and the relative energy deviation $\eta$. Here $\theta$ is roughly the electron coordinates with respect to beam center in unit of $\lambda_{1} / 2 \pi$ where $\lambda_{1}$ is the radiation wavelength. These variables satisfy the well-known pendulum equations. 7

To properly analyze SASE, it is important to account for the discreteness of the electrons. This is achieved by utilizing the Klimontovich distribution function, $\hat{f}, 8$ which 1 s a sum of two parts, the smooth background $\bar{f}$ and the part containing the high frequency fluctuations and modulations, $\bar{f}$. The continuity equation for $\hat{f}$ can likewise be separated into two equations: An equation describing the response of the high-frequency part $\vec{f}$ to the radiation field, and an equation describing the slow, nonlinear evolution of the background distribution $\bar{f}$.

The radiation field is represented by a complex amplitude $a(\nu, x ; z)$, which is the slowly varying part of the full amplitude. $\nu$ is the normalized frequency $\omega / \omega_{1}$, where $\omega_{1}$ is the resonance frequency given by $2 \mathrm{ck}_{\mathrm{u}} \gamma_{0} /\left(1+\mathrm{K}^{2} / 2\right)$. Here $\gamma_{0}$ is the average bean energy in units of $\mathrm{mc}^{2}$ ( $\mathrm{m}$ - electron mass, $\mathrm{c}-$ velocity of $1 \mathrm{ight}$ ), $\mathrm{k}_{\mathrm{u}}=2 \pi / \lambda_{\mathrm{u}}$, $\lambda_{u}$ is the undulator period length, and $k$ is the magnetic deflection parameter. ${ }^{3}$ The wavelength and wave number corresponding to the frequency $\omega_{1}$ are denoted by $\lambda_{1}$ and $k_{1}$, respectively. It is a good approximation to assume that $\nu$ is close to an odd integer, which in 
the following is taken to be $2 \ell+1$. Thus, $\Delta \nu=\nu-2 \ell-1$ is a small quantity. The amplitude satisfies the three-dimensional Maxwell's equation, in which the source term is specified by $\overrightarrow{\mathrm{f}}$.

The continuity equation for $\overline{\hat{f}}$ and the Maxwell's equation form a coupled set. Replacing the slowly varying function $\bar{f}$ by its initial value at $z=0$, these equations become linear, and can be solved explicitly by the method developed by Van kampen. 9 The result for the radiation amplitude in the high-gain limit is

$$
\begin{aligned}
& a(\nu, x ; z)-\frac{A(x) e^{-2 i \mu k_{u} \rho z}}{\int d^{2} y A(y)(1+U(y) d z(\mu) / d \mu)} \\
& \quad \times\left(\int d^{2} y A(y) a(\nu, y ; 0)-i \kappa \sum_{i} \frac{e^{i \theta_{i} \nu} A\left(x_{i}\right)}{\mu+\nu \eta_{i} / \rho}\right) .
\end{aligned}
$$

Here, the summation over $i$ is over all electrons, $\kappa=e$ $\mathrm{K}_{f} / 8 \sqrt{2 \pi} \gamma_{0} \epsilon^{c} \mathrm{k} \rho, c_{0}-$ vacuum dielectric consant, $\mathrm{K}_{\ell}=(-1)^{\ell} \mathrm{k}[\mathrm{JJ}]$, [fJ] is a shorthand expression involving the difference of two Bessel's functions,

$$
\mathrm{z}(\mu)=\rho \int \mathrm{d} \eta \frac{\mathrm{dV}}{\mathrm{d} \eta} \frac{1}{\mu+\nu \eta / \rho}
$$

The mode function $A$ and the complex number $\mu$ are, respectively, the eigenfunction and the eigenvalue of

$$
\left(\mu+\frac{\Delta \nu}{2 \rho}-\frac{1}{2 \rho \mathrm{k}_{\mathrm{u}} \mathrm{k}^{\nu}} \frac{\partial}{\partial \mathrm{x}^{2}}+\mathrm{U}(\mathrm{x}) \mathrm{Z}(\mu)\right) A(\mathrm{x})=0 .
$$

The functions $V$ and $U$ are, respectively, the initial electron distribution in momentum and in transverse coordinates $x$ (normalization: $\left.\int \mathrm{d} \eta \mathrm{V}(\eta)-1, U(0)=1\right)$. The dimensionless parameter $\rho$ in the above plays an important role in characterizing the high-gain behavior and is defined by 1

$$
\rho=\left(\frac{n e^{2} k_{l}^{2}}{32 \sigma_{A} \gamma_{0}^{3} k_{u}^{2} m c^{2} \epsilon_{0}}\right)^{1 / 3}
$$

Here $n$ is the line density of electrons, $\sigma_{A}$ is the cross-sectional area of the electron beam, $\sigma_{A}=\int d^{2} x U(x)$. Equation (3) is essentially that derived and studied earlier by Moore, 10 except that the effects of momentum spread are included. In one dimension it becomes the dispersion relation studied by several authors. 4 There are, in general, a discrete set of complex eigenvalues, as vell as a continuum of real ones. However, the behavior in the high gain limit is governed by eigenvalue $\mu$ with the largest positive imaginary part. In Eq. (1), the term containing the input amplitude a $(; 0)$ describes amplifier mode and represents the solution of the initial-value 
problem in three-dimensional FEL amplification. 10 The term containing the initial electron phases $e^{i \theta_{i} \nu}$ gives gives SASE.

The above results are valid when all electron trajectories are parallel. Taking into account electrons' angular spread, the eigenvalue equation becomes more complicated. ${ }^{6}$ Although the new eigenvalue equation has not been studied in detail, the basic conclusions of this paper, which depend mainly on the fact that there exists an eigenvalue with a positive imaginary part and the associated mode function, will not change upon this generalization.

\section{TRANSVERSE CHARACTERISTICS}

The transverse characteristics of radiation from a high-gain FEL is determined from the structure of $\mathrm{Eq}$. (1). Since the radiation amplitude is specified completely by a single mode function $A(x)$, it follows that the radiation in high gain FEL is guided, as discussed recently, 10,11 The guiding phenomena is important in maintaining high-gain amplification in long undulators; otherwise, the spread of radiation by diffraction would decrease the gain.

It also follows from the explicit form of the solution that the radiation is fully coherent transversely, both in the SASE mode and in the amplifier mode. This result is somewhat surprising for SASE, in comparison with the coherence property of undulator radiation. The latter is, in general, partially coherent transversely, the degree of coherence being determined by the ratio of electron beam phase space area, called the emittance, to the radiation phase-space area.12

The optimum amplifier configuration is obtained when the input radiation amplitude is the complex conjugate of the output mode function $A(x)$. This means in particular that the curvature of the input phase front is of the same magnitude as that of the output but of opposite sign. 13 The symmetry in the phase front geometry is somewhat surprising in view of the extreme asymmetry in the intensity levels.

\section{INTENSITY CHARACTERISTICS}

The power in the radiation is proportional to the ensemble average of $|a|^{2}$. The interference between the terms representing SASE and the radiation produced in the amplifier mode clearly vanishes, and the ensemble average of the SASE term can readily be performed assuming that electrons are not correlated initially. The intensity growth and the spectral characteristics are determined mainly by the imaginary part $\mu I$ of $\mu$. In this way, one obtains the power spectrum,

$$
\frac{d P}{d \omega}=e^{\tau} s\left(\Delta \omega / \omega_{m}\right)\left[g_{A}\left(\frac{d P}{d \omega}\right)_{0}+g_{s} \frac{\rho E_{0}}{2 \pi}\right] \text {, }
$$

where $\tau-8 \pi \mu_{I}{ }^{m} \rho \mathrm{N}, \mu_{I}^{m}$ the maximum the value of $\mu_{I}$ as a function of $\Delta \nu, \Delta \omega=\omega-\omega_{m}, \omega_{m}-I$ the frequency at maximum growth, $s(x)=$ exp $\left(-x^{2} / 2 \sigma_{\Delta}{ }^{2}\right)$, and $g_{A}$ and $g_{S}$ are quantities of order unity. The first term in Eq. (5) gives the power spectrum for an FEL operating in the amplifier mode, and one finds the growth of the input power spectrum 
$(d P / d w)_{0}$ to be exponential. The power spectrum for SASE is given by the second term, which exhibits the same exponential growth: with the input replaced by the effective noise power spectrum

$$
\left[\frac{d P}{d \omega}\right)_{0, S A S E}=\frac{\rho E_{0}}{2 \pi} .
$$

where $E_{O}$ is the average beam energy. The function $S$ describes the frequency dependence of the gain for coherent amplification, as well as the spectral shape of the SASE radiation. In one dimension, for zero momertum spread, one obtains $g_{A}=g_{S}=1 / 9$ and the bandwidth

$$
\sigma_{\Delta}=(9 \rho / 2 \pi \sqrt{3} \mathrm{~N})^{\frac{1}{2}} \text {. }
$$

For momentum spread much larger than $\rho$, the eigenvalue $\mu$ is real and there is no exponential growth. Therefore, for exponential growth to occur.

$$
\sigma_{\eta} \leq \rho,
$$

where $\sigma$ is the relative momentum spread in the electron beam. The total SASE power, obtained by integrating over the frequency, is

$$
\mathrm{P}_{\text {SASE }}=\rho \mathrm{P}_{\text {beam }} \mathrm{g}_{\mathrm{S}} \mathrm{e}^{\tau} / \mathrm{N}_{\ell \mathrm{C}}
$$

where $\mathrm{P}_{\text {beam }}$ is the kinetic power in the beam (equal to $\mathrm{E}_{\mathrm{o}} \mathrm{I} / \mathrm{e}$, where $I$ - beam current) and $N_{\ell c}-n \lambda_{1}(2 \pi)^{-\frac{\pi}{2}} / \sigma_{\Delta}$ is the number of electrons in one coherence length.

The slow variation of $\bar{f}$ with respect to $z$ is determined by a procedure known as the quasi-linear approximation in plasma physics. 14 From the reiulting nonlinear Fokker-Planck equation, one finds that the average value of $\eta$ must decrease so as to conserve the total energy of the radiation-beam gystem. In addition the rms spread of $\eta$, $\sigma_{\eta}$, is found to increase as $\sigma_{\eta}^{2} \approx \rho^{2} \mathrm{~g}_{\mathrm{s}} \mathrm{e}^{\tau} / \mathrm{N} l \mathrm{c}$. Since the growth rate becomes negligible when $\sigma \gg{ }^{\eta} \rho$, the exponential growth will stop when the factor $g_{s} \mathrm{e}^{\tau}$ becomes about $\mathrm{N}_{\ell c} \cdot{ }^{15}$ In view of Eq. (9) the power at saturation becomes.

$$
\mathrm{P}_{\text {sat }} \sim \rho \mathrm{P}_{\text {beam }}
$$

The saturation occurs when $\rho \mathrm{N} \approx \ln \left(\mathrm{N}_{\ell \mathrm{c}} / \mathrm{g}_{\mathrm{S}}\right) / 8 \pi \mu_{I}^{\mathrm{m}}$. For parameters considered here this becomes ${ }^{1}$

$$
N-1 / \rho
$$

In view of Eq. (7), the bandwidth at saturation is $\omega / \Delta \omega-N$, which is the same as the bandwidth of the spontaneous radiation from an undula. tor with the same $N$. The crucial importance of the parameter $\rho$ in determining high-gain behavior should be clear by now: It enters into the growth rate [Eq. (5) j, the condition for efficiency [Eq. (8)], the effective noise power [Eq. (6)], the bandwidth [Eq. (7)], the saturation condition [Eq. (11)], and the saturated power [Eq. (10)]. 
Figure 1 shows data from the microwave FEL experiment at Lawrence Livermore National Laboratory. 2 For this experiment, the onedimensional theory is appropriate, and the growth rate is calculated to be $42.1 \mathrm{~dB} / \mathrm{m}$. The observed growth rate was $35 \mathrm{~dB} / \mathrm{m}$; the discrepancy may be due to space-charge effects. Taking the observed growth rate and computing the coefficient in Eq. (9), one obtains the dotted line ir Fig. 1. The agreement is encouraging.

\section{v. A HIGH-GAIN FEL ON A BY-PASS OF A. STORAGE RING}

Modern storage rings provide the high-density electron beams required for high-gain FEL operation. For efficient interaction, the undulator must be long and have a narrow gap. Such a device, if placed in a normal section of a storage ring, would severely limit the acceptance of the ring and thus reduce the beam lifetime due to scattering with the residual gas. Moreover, the interaction of the beam with an FEL undulator is disruptive to the beam itself in terms of energy loss and increased momentum spread. To avoid these problems, the undulator can be placed in a special by-pass section, 16 as shown schematically in Fig. 2. The electron beam normally circulates in the storage ring without passing chrough the FEL undulator. Once every damping time, the beam is directed into the by-pass section, where the interaction with the undulator takes place, generating intense, coherent radiation. As the beam leaves the undulator, it is deflected back into the storage ring, where synchrotron radiation damping reduces the induced energy spread. After one damping time, the beam is ready to be injected into the by-pass again.

To relate the parameter $a$ to the electron beam and storage ring parameters, we assume that the electron beans in the undulator are focused with the effective $\beta$ nction given by

$$
\beta_{\mathrm{x}}-\beta_{\mathrm{y}}-\frac{\lambda_{\mathrm{u}} \gamma_{0}}{\pi \mathrm{K}}
$$

Equation (4) can then be written as follows: 17

$$
\rho-\left(\frac{1}{16 \pi} \frac{r_{e}}{e c} \frac{k^{3}[J J]^{2}}{2\left(1+k^{2} / 2\right)} \frac{\lambda_{1}}{\gamma_{0}^{2}} \frac{I_{p}}{\sqrt{\epsilon x^{\epsilon} y}}\right)^{1 / 3}
$$

Here, we assume operation at the fundamental harmonic, $l=1$. In the above, $r_{e}$ is the classical electron radius, $I_{p}$ is the peak current, and $\epsilon$ and $\epsilon$ are, respectively, the electron emittances in the hor-
izontâl and the vertical directions.

Equation (13) gives the general criteria for design optimization. The peak current should be large, the emittances small. Low electron energy is preferable for a given wavelength $\lambda_{1}$. Also, a large $K$ is preferred, leading to an undulater of small magnet gap.

A feasibility study of a storage ring for a high gain FEL operation at $400 \AA$ was recently carried out at Lawrence Berkeley Laboratory. 18 Important aspects of storage ring issues, such as collective 
instabilities and lattice optimization, by-pass considerations, and operational requirements were studied. The general conclusions were that the high beam quality demanded for these high-gain FEL can be achieved with presently available accelerator technology. Among five different storage rings considered, one with a circumference of $144 \mathrm{~m}$, based on the combined-function lattice, looks most promising. Running at $750 \mathrm{MeV}$, the horizontal emittance is $1 \times 10^{-8} \mathrm{~m}-\mathrm{rad}$, with a peak current of $200 \mathrm{~A}$ and a momentum spread of $0.2 \%$. The undulator in the by-pass is of the permanent magnet-steel hybrid type, $1920 \mathrm{~m}$ in length, and has a period of $2.29 \mathrm{~cm}$ with a $3-\mathrm{mm}$ magnet gap. Such a system will produce tens of megawatts of $400 \AA$ radiation, fully coherent transversely, with about a 0.18 relative bandwidth. The radiation is generated in pulses about 100 ps in duration with a repetition rate of about $20 \mathrm{~Hz}$.

\section{HIGH-GAIN FELS BASED ON LINACS}

Linacs are more compact than storage rings. However, the emittances and energy spread of electron beams in linacs tend to be larger than those in storage rings. The drawback is removed if the electron beams from a linac are routed to a damping ring, which is basically a compact storage ring whose sole function is to decrease the beam emit. tances and the energy spread by radiation damping. A system consisting of a linac, a damping ring, and a long undulator offers another approach to a high-gain FEL. Such a system is currently being studied at Brookhaven National Laboratory. A $750-\mathrm{MeV}$ linac has a total length about 50 to $60 \mathrm{~m}$, which is also about the circuriference of the damping ring.

\section{REFERENCES}

1. B. Bonifacio, N. Narducci, and C. Pellegrini, Opt. Comm. 50, 373 (1984).

2. T.J. Orzechowski et al., Phys. Rev. Lett. $\underline{54}, 889$ (1985).

3. See for example, S. Krinsky, IEEE Trans. Nucl. Sci., NS-30, 3078 (1983).

4. N.M. Kroll and W.A. MeMullin, Phys. Rev. A17, 300 (1978); A. Gover, and Z. Livini, Opt. Comm. 26, 375 (1978).

5. K.-J. Kim, Proc. 7th Int. FEL Conf., Lake Tahoe, CA. Sept. 1985. See also J.M.. Wang and L.H. Yu, ibid.

6. K.-J. Kim, Three dimensional analysis of coherent amplification and Self-Amplified Spontaneous Emission in Free Electron Lasers, LBL-21426 (April 1986), submitted to Phys. Rev. Lett. SASE for electron beams of infinite transverse extent was recently studied by L.H. Yu and S. Krirsky, these proceedings.

7. For a review see, W.B. Colson, IEEE J. Quant. Elect. QE17, 1417 (1981).

8. Y.L. Klimontovich, Soviet Phys. JETP 6, 753 (1958). See also S. Ichimaru, Basic Principle of Plasma Physics (W.A. Bejamin, Inc., 1973). Appendix A. 
9. N.G. Van Kampen, -Physica 21, 949 (1951); K.M. Case, Ann. Phys. I. 349 (1959).

10. G.T. Moore, Opt. Comm. 54, 121 (1985).

11. E.T. Scharlemann, A.M. Sessler, J.S. Wurtele, Phys. Rev. Lett. 54. 1925 (1985).

12. K.J. Kim, preprint LBL-20181 (July, 1985), to be published in Nucl. Instr. Method.

13. This was independently noticed by G.T. Moore, University of New Mexico preprint.

14. See for example, G. Schmidt, Physics of High Temperature Plasma (Academic Press, New York, 1979), section 9-2. The method was applied to 1-D FEL amplifier problem by T. Taguchi, K. Mima and T. Mochizuki, Phys, Rev. Lett., 46, 824 (1981).

15. This result was first derived by $S$. Krinsky using the fluctuation dissipation theorem (BNL-preprint).

16. J. Murphy and C. Pellegrini, J. Opt. Soc. Am. 1B, 530 (1984).

17. K-J. Kim, J.J. Bisognano, A.A. Garren, K. Halbach, and J.M. Peterson, Nuc1. Instr. Methods, A239, 54 (1985).

18. J.J. Bisognano et al., preprint LBL-19771 (March, 1985), submitted to Part. Acc.

19. K. Halbach, Journal de Physique Colloque, C1, 44, 211 (1983). 


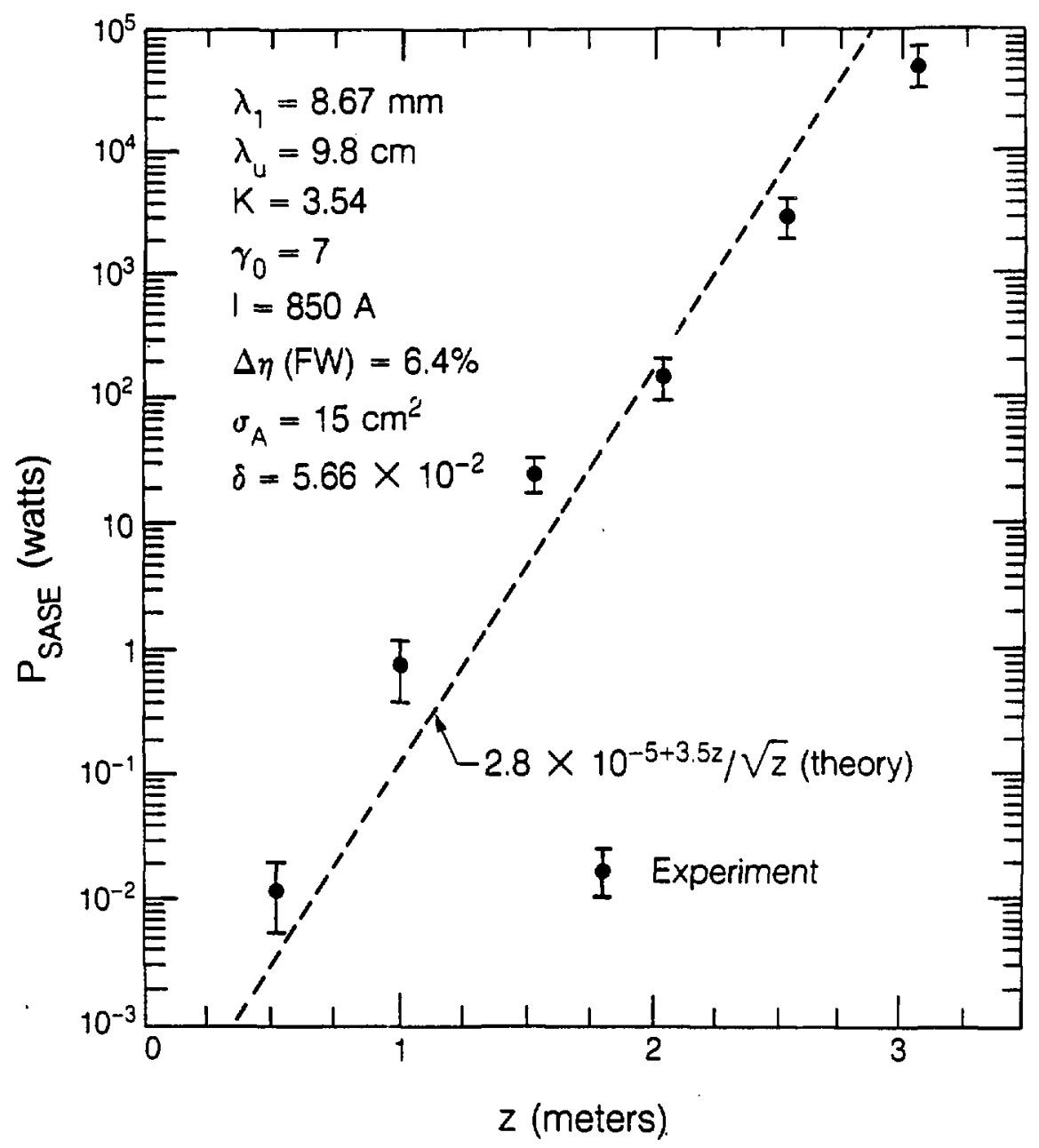

XBL 864-10398

Figure 1. Data from Ref. 2 (courtesy of $T$. Orzechowski) compared with the prediction. 


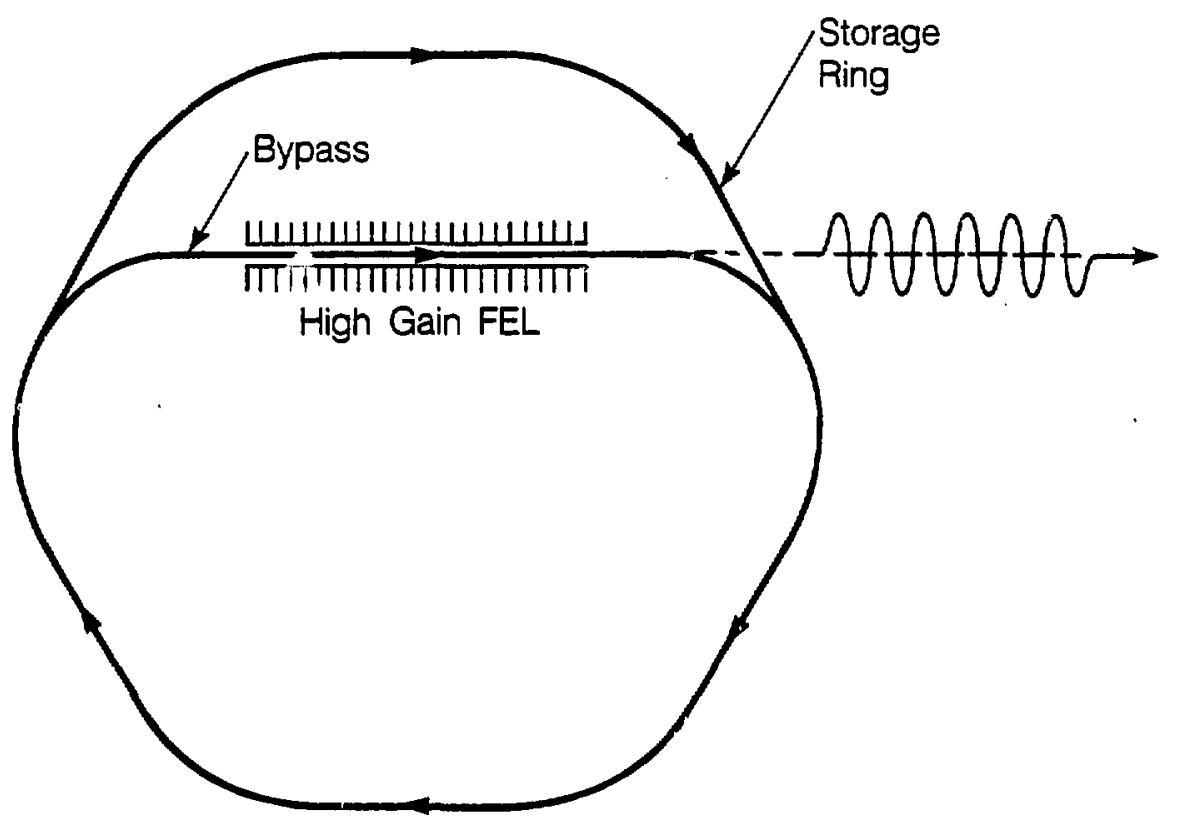

XBL 854-1017!

Figure 2. Schematic drawing of a storage ring with a bypass for a high-gain FEL. 